\title{
P20-2 I LB. Gene-to-gene differences in evolutionary rate between HIV-I and natural SIV from sooty mangabeys: implications for vaccine tests in non-human primates
}

\author{
W Fischer*1, C Apetrei ${ }^{2}$, BH Hahn ${ }^{3}$, NL Letvin ${ }^{4}$, GJ Nabel ${ }^{5}$ and BT Korber ${ }^{1}$
}

Address: ${ }^{1}$ Los Alamos National Laboratory, Los Alamos, NM, USA, ${ }^{2}$ Department of Tropical Medicine, Tulane University, New Orleans, LA, USA, ${ }^{3}$ Depts. of Medicine and Microbiology, Univ. of Alabama at Birmingham, Birmingham, AL, USA, ${ }^{4}$ Beth Israel Deaconess Medical Center, Boston, MA, USA and ${ }^{5}$ Vaccine Research Center, NIH/NAID, Bethesda, MD, USA

* Corresponding author

from AIDS Vaccine 2009

Paris, France. 19-22 October 2009

Published: 22 October 2009

Retrovirology 2009, 6(Suppl 3):P425 doi:10.1 186/1742-4690-6-S3-P425

This abstract is available from: http://www.retrovirology.com/content/6/S3/P425

(C) 2009 Fischer et al; licensee BioMed Central Ltd.

\section{Background}

The most useful model system for HIV vaccines is SIV infection of non-human primates (typically rhesus macaques). The pathogenic SIV strains in common use were derived from macaques infected in captivity by viruses originally from sooty mangabeys. In the course of developing new viral stocks and vaccine antigens for challenge studies, we investigated the mode of evolution in natural SIV isolates from sooty mangabeys, comparing it to that of HIV-1 isolates from the global pandemic.

\section{Methods}

We assembled a diverse SIV set comprising sooty mangabey SIV $\left(\operatorname{SIV}_{\text {smm }}\right)$ whole-genome sequences from published and unpublished sources, including novel sequences; all sequences were either directly isolated or minimally passaged. Diversity-matched HIV-1 data sets were constructed for HIV-1 M-group sequences for four vaccine candidate genes, Gag, Pol, Env, and Nef. We constructed phylogenetic trees, and calculated substitution rate ratios to infer selective pressure upon the two different viruses in the two different primate hosts.

\section{Results}

The ratios of synonymous to non-synonymous substitutions $\left(D_{n} / D_{s}\right)$ for Gag, Pol, Env, and Nef) were 1.4 to 2.0 times higher for HIV-1 than for the $\mathrm{SIV}_{\text {smm }}$. The greatest disparities were observed in HIV-1 Gag and Pol. In both these genes, SIV $_{\text {smm }}$ and HIV-1 have similar numbers of amino-acid changes, but $\operatorname{SIV}_{\text {smm }}$ has a higher number of synonymous substitutions. In constrast, non-synonymous substitutions are elevated in HIV-1 Env relative to SIV $_{\text {smm }^{\prime}}$ and HIV-1 Nef has a higher rate of both synonymous and non-synonymous substitutions.

\section{Conclusion}

HIV-1 in humans is under stronger diversifying selection in general than is SIV $_{\text {smm }}$ in sooty mangabeys. This disparity is strikingly pronounced in Env, possibly due to greater immune pressure in humans this has implications for studying the impact of vaccine-elicited immune responses on pathogenesis and protection, and the relevance of macaque vaccine studies to humans. 